\title{
Complexity of the Szeged index, edge orbits, and some nanotubical fullerenes
}

\author{
Yaser Alizadeh $^{1}$ (1), Sandi Klavžar*2 ${ }^{*}$ \\ ${ }^{1}$ Department of Mathematics, Hakim Sabzevari University, Sabzevar, Iran \\ ${ }^{2}$ Faculty of Mathematics and Physics, University of Ljubljana, Slovenia \& Faculty of Natural Sciences \\ and Mathematics, University of Maribor, Slovenia \& Institute of Mathematics, Physics and Mechanics, \\ Ljubljana, Slovenia
}

\begin{abstract}
Let $I$ be a summation-type topological index. The $I$-complexity $C_{I}(G)$ of a graph $G$ is the number of different contributions to $I(G)$ in its summation formula. In this paper the complexity $C_{\mathrm{Sz}}(G)$ is investigated, where $\mathrm{Sz}$ is the well-studied Szeged index. Let $O_{e}(G)$ (resp. $O_{v}(G)$ ) be the number of edge (resp. vertex) orbits of $G$. While $C_{\mathrm{Sz}}(G) \leq O_{e}(G)$ holds for any graph $G$, it is shown that for any $m \geq 1$ there exists a vertex-transitive graph $G_{m}$ with $C_{\mathrm{Sz}}\left(G_{m}\right)=O_{e}\left(G_{m}\right)=m$. Also, for any $1 \leq k \leq m+1$ there exists a graph $G_{m, k}$ with $C_{\mathrm{Sz}}\left(G_{m, k}\right)=O_{e}\left(G_{m, k}\right)=m$ and $C_{W}\left(G_{m, k}\right)=O_{v}\left(G_{m, k}\right)=k$. The Sz-complexity is determined for a family of $(5,0)$-nanotubical fullerenes and the Szeged index is compared with the total eccentricity.
\end{abstract}

Mathematics Subject Classification (2010). 05C12, 05C25, 92E10

Keywords. Szeged index, Szeged complexity, vertex-transitive graph, edge-transitive graphs, fullerene, total eccentricity

\section{Introduction}

In mathematical chemistry, any function which assigns a real number to a (chemical) graph and is invariant under graph isomorphism is called a topological index. Of course, one has countless possibilities how to define a (new) topological index; hence it is important to design it to be applicable and, also not negligible, mathematically appealing. The reader is invited to books $[16,17,31]$ for examples of topological indices that have passed these requirements. We also refer to $[13,28]$ for a couple of recent chemical applications, to [7] for a recent investigation of several infinite convex benzenoid networks via numerous topological indices, as well as to [19] for studies of additional topological indices on hexderived networks.

In this paper we are primarily interested in the Szeged index that turned out to be one of the relevant topological indices. It was introduced in [15] and proved to be chemically relevant in $[8,20]$ as well as in [7], where Szeged-like indices are involved in the investigation. The papers $[18,25]$ present a couple of recent developments on the Szeged index.

\footnotetext{
*Corresponding Author.

Email addresses: y.alizadeh@hsu.ac.ir (Y. Alizadeh), sandi.klavzar@fmf.uni-lj.si (S. Klavžar)

Received: 11.02.2017; Accepted: 17.09.2018
} 

form

Let $G=(V(G), E(G))$ be a graph. Suppose that a topological index $I$ either of the

$$
I(G)=\sum_{v \in V(G)} f(v),
$$

or of the form

$$
I(G)=\sum_{e \in E(G)} f(e),
$$

where $f: V(G) \rightarrow \mathbb{R}$ (resp. $f: E(G) \rightarrow \mathbb{R}$ ) is a real function. The most striking example is obtained by setting $f(v)$ to be the sum of the distances from $v$ to all the other vertices, because in this case (1.1) reads as $I(G)=2 W(G)$, where $W(G)$ is the Wiener index of $G$. Vertices $u$ and $u^{\prime}$ (resp. edges $e$ and $e^{\prime}$ ) are in relation $\sim_{I}$ if $f(u)=f\left(u^{\prime}\right)$ (resp. $f(e)=f\left(e^{\prime}\right)$ ). Clearly, $\sim_{I}$ is an equivalence relation. Let $V(G) / \sim_{I}=\left\{V_{1}, \ldots, V_{k}\right\}$ (resp. $E(G) / \sim_{I}=\left\{E_{1}, \ldots, E_{k}\right\}$ ) be its equivalence classes and let $v_{i} \in V_{i}$ (resp. $e_{i} \in E_{i}$ ) for $i \in[k]=\{1, \ldots, k\}$. Then (1.1) can be rewritten as

$$
I(G)=\sum_{i=1}^{k}\left|V_{i}\right| f\left(v_{i}\right),
$$

while (1.2) reduces to

$$
I(G)=\sum_{i=1}^{k}\left|E_{i}\right| f\left(e_{i}\right) .
$$

The value $k$ is called the $I$-complexity of $G$ and denoted $C_{I}(G)$. This concept was introduced in [4] and studied on the connective eccentricity index; see [14,32] for more information on the latter index. Earlier, the complexity with respect to the Wiener index was investigated in [3] under the name Wiener dimension. For recent developments on the Wiener complexity see $[1,5,21]$. In addition, in [2] the Szeged and the $P I_{v}$ dimension was investigated.

The paper is organized as follows. In the next section additional concepts needed are introduced. In the central part of the paper, Section 3, the Sz-complexity of graphs is compared to the numbers of their edge orbits $O_{e}$ and the number of their vertex orbits $O_{v}$. It is observed that for any graph $G, C_{\mathrm{Sz}}(G) \leq O_{e}(G)$, and demonstrated that for any integer $m \geq 1$ there exists a vertex-transitive graph $G_{m}$ with $C_{\mathrm{Sz}}\left(G_{m}\right)=O_{e}\left(G_{m}\right)=m$. Moreover, for any $1 \leq k \leq m+1$ there exists a graph $G_{m, k}$ with $C_{\mathrm{Sz}}\left(G_{m, k}\right)=O_{e}\left(G_{m, k}\right)=$ $m$ and $C_{W}\left(G_{m, k}\right)=O_{v}\left(G_{m, k}\right)=k$. In Section 4 the Sz-complexity is determined for a family of $(5,0)$-nanotubical fullerenes. As a consequence the Szeged index of these fullerenes is determined. In the final section it is proved that if $G$ is a connected graph of order at least 4, then $\operatorname{Sz}(G) \geq \operatorname{Ecc}(G)$, where equality holds if and only if $G=P_{4}$.

\section{Preliminaries}

All graphs considered in this paper are connected. The degree of a vertex $u$ of a graph $G$ is denoted with $\operatorname{deg}_{G}(u)$. The distance between vertices $u$ and $v$ of a graph $G$ is denoted by $d_{G}(u, v)$. The distance $d_{G}(v)$ of vertex $v$ is defined as $d_{G}(v)=\sum_{u \in V(G)} d_{G}(v, u)$. The eccentricity $\operatorname{ecc}_{G}(v)$ of $v$ is the largest distance between $v$ and the vertices of $G$. Whenever $G$ is clear form the context, we may omit the index $G$ in the above notation. The maximum and the minimum eccentricity among all vertices of $G$ are the diameter $\operatorname{diam}(G)$ and the radius $\operatorname{rad}(G)$, respectively.

The Wiener index of graph $G$ is the sum of distances between all pairs of vertices of $G$, that is,

$$
W(G)=\frac{1}{2} \sum_{v \in V(G)} d(v) .
$$


Let $e=u v$ be an edge of graph $G$. The number of vertices of $G$ lying closer to $u$ than to $v$ is denoted by $n_{u}(e)$. Analogously, $n_{v}(e)$ is the number of vertices of $G$ lying closer to $v$ than to $u$. The Szeged index [15] of $G$ is defined with

$$
\mathrm{Sz}(G)=\sum_{e=u v \in E(G)} n_{u}(e) n_{v}(e) .
$$

The total eccentricity of $G$ is defined as

$$
\operatorname{Ecc}(G)=\sum_{v \in V(G)} \operatorname{ecc}(v) .
$$

Let $\operatorname{Aut}(G)$ denote the automorphism group of $G$. A graph $G$ is vertex-transitive if two any vertices $u$ and $v$ of $G$ there exists $\alpha \in \operatorname{Aut}(G)$ such that $\alpha(u)=v$. Let $O_{v}(G)$ and $O_{e}(G)$ be the number of vertex orbits and edge orbits of $G$ under the action of $\operatorname{Aut}(G)$, respectively. A function $f: V(G) \rightarrow \mathbb{R}$ (resp. $f: E(G) \rightarrow \mathbb{R}$ ) is a graph function if it is invariant under automorphisms of $G$; that is, if $\alpha \in \operatorname{Aut}(G)$ and $u$ and $u^{\prime}$ belong to the same vertex orbit (resp. $e$ and $e^{\prime}$ belong to the same edge orbit), then $f(u)=f\left(u^{\prime}\right)$ (resp. $\left.f(e)=f\left(e^{\prime}\right)\right)$.

We will use the following result that goes back to [12].

Proposition 2.1. If $e=u v$ is an edge of a connected graph $G$, then $d_{G}(u)-d_{G}(v)=$ $n_{v}(e)-n_{u}(e)$.

\section{Sz-complexity versus number of edge orbits}

Suppose that a topological index $I$ is of the form (1.1) or (1.2), where $f$ is a graph function. Then by definition,

$$
C_{I}(G) \leq O_{v}(G) \quad\left(\operatorname{resp} . C_{I}(G) \leq O_{e}(G)\right) .
$$

We note in passing that if $C_{I}(G) \leq O_{v}(G)$, then $C_{I}(G) \leq O_{e}(G)+1$. This follows from a result of Buset [9] asserting that if $G$ is a (connected) graph, then $O_{v}(G) \leq O_{e}(G)+1$.

Theorem 3.1. If $G$ is a graph, then $C_{\mathrm{Sz}}(G) \leq O_{e}(G)$. Moreover, for any integer $m \geq 1$ there exists a vertex-transitive graph $G_{m}$ with $C_{\mathrm{Sz}}\left(G_{m}\right)=O_{e}\left(G_{m}\right)=m$.

Proof. For the first assertion, in view of (3.1) it suffices to prove that the function $f(e)=$ $n_{u}(e) n_{v}(e)$, where $e=u v \in E(G)$, is a graph function. Hence let $e=u v$ and $e^{\prime}=x y$ be edges from the same edge orbit, and let $\alpha \in \operatorname{Aut}(G)$ such that $\alpha(u)=x$ and $\alpha(v)=y$. Let $\Gamma_{k}(u)=\{w \in V(G) \mid d(u, w)=k\}, 0 \leq k \leq \operatorname{ecc}(u)$. We claim that $n_{u}(e)=n_{x}\left(e^{\prime}\right)$ and $n_{v}(e)=n_{y}\left(e^{\prime}\right)$. For this sake observe first that $n_{u}(u v)=\sum_{k=0}^{\operatorname{ecc}(u)}\left|\Gamma_{k}(u) \cap \Gamma_{k+1}(v)\right|$. Furthermore,

$$
w \in \Gamma_{k}(u) \bigcap \Gamma_{k+1}(v) \Leftrightarrow \alpha(w) \in \Gamma_{k}(\alpha(u)) \bigcap \Gamma_{k+1}(\alpha(v))=\Gamma_{k}(x) \bigcap \Gamma_{k+1}(y) .
$$

Therefore

$$
n_{u}(u v)=\sum_{k=0}^{\operatorname{ecc}(u)}\left|\Gamma_{k}(u) \bigcap \Gamma_{k+1}(v)\right|=\sum_{k=0}^{\operatorname{ecc}(x)}\left|\Gamma_{k}(x) \bigcap \Gamma_{k+1}(y)\right|=n_{x}(x y) .
$$

This proves the first assertion of the theorem.

Let $m \geq 1$ and set $n=3 m+1$. Define the graph $G_{m}$ on the vertex set $[n]_{0}=$ $\{0,1, \ldots, n-1\}$, where the vertex $i$ is adjacent to vertices $i \pm 1, \ldots, i \pm m(\bmod n)$. Clearly, $G_{m}$ is vertex-transitive. (Alternatively, $G_{m}$ belongs to the family of circulant graphs which are well-known to be vertex-transitive.) Consider now the edge $i j$, where $j=i \pm k$, $1 \leq k \leq m$. Then $i j$ lies in precisely $m-k+1$ complete subgraphs $K_{m+1}$. It follows that $G_{m}$ contains at least $m$ edge orbits. On the other hand, any two edges that differ by the same integer, are in the same edge orbit. We conclude that $O_{e}\left(G_{m}\right)=m$. Finally, any pair of vertices has a common adjacent vertex, hence $\operatorname{diam}\left(G_{m}\right)=2$. Moreover, since $G_{m}$ 
is (2m)-regular, vertices $i$ and $j=i \pm k, 1 \leq k \leq m$, have exactly $2 m-k-1$ common adjacent vertices. Consequently $n_{i}(i j)=n_{j}(i j)=k+1$. Therefore, $C_{\mathrm{Sz}}\left(G_{m}\right)=m$.

In view of (3.1) the reader might wonder why the first assertion of Theorem 3.1 requires a proof. To see that the assertion need not be true in general, consider the following example. Let $G$ be a graph with the vertex set $[n]_{0}$, and define the invariant $\operatorname{Sz}^{\prime}(G)=$ $\sum_{e=i j \in E(G)}\left(n_{i}(e)-n_{j}(e)\right)$. Then in general $C_{\mathrm{Sz}^{\prime}}(G) \leq O_{e}(G)$ does not hold. For a small example consider the path $P_{3}$ of order 3. Clearly, $O_{e}\left(P_{3}\right)=1$, but $C_{\mathrm{Sz}^{\prime}}\left(P_{3}\right)=2$.

In Theorem 3.1 we have seen that there exist graphs with a single vertex orbit and with an arbitrary large Sz-complexity. On the other hand, we also have:

Proposition 3.2. There exists an infinite family of non edge-transitive graphs with Szcomplexity equal to 1.

Proof. Let $W_{n}, n \geq 3$, be the $n$-wheel, that is, the graph obtained from the $n$-cycle $C_{n}$ and an extra vertex joined to all the vertices of the cycle. The cogwheel $M_{n}$ is then obtained from $W_{n}$ by subdividing each edge of $C_{n}$ by one vertex. Clearly, $M_{n}$ is not edge-transitive. On the other hand it is straightforward to infer that for any edge $e=u v \in E\left(M_{n}\right)$ we have $\left\{n_{u}(e), n_{v}(e)\right\}=\{3,2 n-2\}$, so that $C_{\mathrm{Sz}}\left(M_{n}\right)=1$.

It is straightforward to see that the cogwheel $M_{n}$ has two edge orbits. Hence an interesting question appears whether there exist (infinite families of) graphs with Sz-complexity equal to 1 and with an arbitrary number of edge orbits.

By the above-mentioned Buset's result [9] we have $O_{v}(G) \leq O_{e}(G)+1$. We next show that comparing the Sz-complexity with the W-complexity anything else can happen. More precisely:

Theorem 3.3. If $m \geq 1$ and $1 \leq k \leq m+1$, then there exists a graph $G_{m, k}$ with $C_{\mathrm{Sz}}\left(G_{m, k}\right)=O_{e}\left(G_{m, k}\right)=m$ and $C_{W}\left(G_{m, k}\right)=O_{v}\left(G_{m, k}\right)=k$.

Proof. Let $m \geq 1$ and set $G_{m, m+1}=P_{2 m+1}$. Then it can be routinely checked that $C_{\mathrm{Sz}}\left(G_{m, m+1}\right)=O_{e}\left(G_{m, m+1}\right)=m$ and $C_{W}\left(G_{m, m+1}\right)=O_{v}\left(G_{m, m+1}\right)=m+1$.

Suppose in the rest that $m \geq 1$ and $1 \leq k \leq m$. Let $G_{m}$ be the graph of order $3 m+1$ as constructed in the proof of Theorem 3.1, where $V\left(G_{m}\right)=[3 m+1]_{0}$. Let $G_{m, k}$ be the graph obtained from a disjoint union of $G_{m-k+1}$ and $m-k+1$ copies of the path $P_{k}$, by identifying each of the vertices of $G_{m-k+1}$ with an end-vertex of a respective copy of $P_{k}$. The construction is illustrated in Fig. 1 with the graph $G_{5,4}$. Note also that $G_{m, 1}=G_{m}$.

As already inferred in the proof of Theorem 3.1, $O_{e}\left(G_{m-k+1}\right)=m-k+1$. This in turn implies that $O_{e}\left(G_{m, k}\right)=(m-k+1)+(k-1)=m$. Consider now the edges $0 j, j \in[m-k+1]$, of $G_{m-k+1}$ (considered as a subgraph of $G_{m, k}$ ), and observe that $a_{j}=n_{0}(0 j)=n_{j}(0 j)=(j+1) k$. Furthermore, for the $k-1$ edges $e_{i}$ of an arbitrary fixed attached $P_{k}$, we easily infer that their contributions to the Szeged index are $b_{i}=$ $i(3 k(m-k+1)+k-i), i \in[k-1]$, because $\left|V\left(G_{m, k}\right)\right|=3 k(m-k+1)+k$. Since for any $i \in[k-1]$ and any $j \in[m-k+1]$ we have $i(3 k(m-k+1)+k-i)>(j+1) k$, there exist $(m-k+1)+(k-1)=m$ different contributions to $\operatorname{Sz}\left(G_{m, k}\right)$. Hence in view of the second assertion of (3.1) and the above fact that $O_{e}\left(G_{m, k}\right)=m$ we conclude that $C_{\mathrm{Sz}}\left(G_{m, k}\right)=m$.

Since $G_{m-k+1}$ is vertex-transitive, it follows directly that $O_{v}\left(G_{m, k}\right)=k$. Moreover, by symmetry, all the vertices of $G_{m-k+1}$ (considered as vertices of $G_{m, k}$ ) have the same distance in $G_{m, k}$. Consider now the vertices of an arbitrary fixed $P_{k}$ subgraph of $G_{m, k}$. Then by iterative applications of Proposition 2.1 we get that all these $k$ vertices have different distances. Hence by the first assertion of (3.1) we conclude that $C_{W}\left(G_{m, k}\right)=$ $k$. 


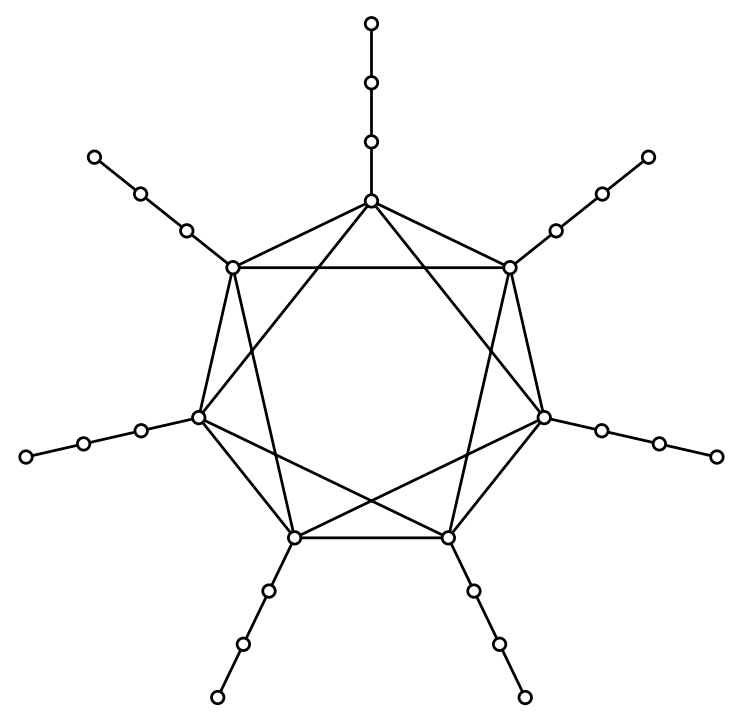

Figure 1. The graph $G_{5,4}$

Considering the graphs from the proof of Theorem 3.3 and noting that $C_{\text {Ecc }}\left(P_{2 m+1}\right)=$ $m+1$ for $m \geq 1$ and that $C_{\mathrm{Ecc}}\left(G_{m, k}\right)=k$ for $m \geq 1$ and $1 \leq k \leq m$, we get in passing the following result.

Corollary 3.4. If $m \geq 1$ and $1 \leq k \leq m+1$, then there exists a graph $G_{m, k}$ with $C_{\mathrm{Sz}}\left(G_{m, k}\right)=O_{e}\left(G_{m, k}\right)=m$ and $C_{\mathrm{Ecc}}\left(G_{m, k}\right)=O_{v}\left(G_{m, k}\right)=k$.

\section{Sz-complexity of the fullerenes $C_{10 n}$}

In this section we compute the Sz-complexity of a family of fullerene graphs. This yields another infinite family of (chemical) graphs for which the Sz-complexity coincides with the number of edge orbits.

A fullerene graph is a 3-connected, 3-regular plane graph with only pentagonal and hexagonal faces. They have exactly twelve pentagonal faces. Here we consider the family of fullerenes $C_{10 n}, n \geq 2$, known as $(5,0)$-nanotubical fullerenes. (For distance properties of $C_{10 n}$ see $[3,6]$.) The fullerene $C_{10 n}$ contains $10 n$ vertices which are grouped into $n+1$ layers $L_{0}, L_{1}, \ldots, L_{n}$, where the layers $L_{0}$ and $L_{n}$ contain 5 vertices, while each of the other layers contains 10 vertices. In Fig. 2 the case $n=5$ is drawn, that is, $C_{50}$, from which the general edge structure of these graphs should be clear.

The main result of this section reads as follows.

Theorem 4.1. If $n \geq 3$, then $C_{\mathrm{Sz}}\left(C_{10 n}\right)=n+1=O_{e}\left(C_{10 n}\right)$.

Proof. Let $L_{0}, L_{1}, \ldots, L_{n}$ be the layers of vertices of $C_{10 n}$, and let $S_{i}$ be the set of edges connecting a vertex of $L_{i-1}$ to a vertex of $L_{i}$. It is not difficult to observe that for $0 \leq i \leq\left\lfloor\frac{n+1}{2}\right\rfloor$, the edges of $L_{i}$ and $L_{n-i}$ are in the same orbit and that for $1 \leq j \leq\left\lfloor\frac{n}{2}\right\rfloor$, the edges of $S_{j}$ and edges of $S_{n-j}$ are in the same edge orbit. Hence, using Theorem 3.1, $C_{\mathrm{Sz}}\left(C_{10 n}\right) \leq O_{e}\left(C_{10 n}\right)=n+1$.

We checked by computer that $C_{\mathrm{Sz}}\left(C_{10 n}\right)=n+1$ holds for $3 \leq n \leq 9$. In the rest we prove by induction that the same holds for $n \geq 9$. To simplify the notation, set $N(e)=n_{u}(e) n_{v}(e)$ for an edge $e=u v$. For $n=9,10$, let $e_{i} \in L_{i}, 0 \leq i \leq\left\lfloor\frac{n}{2}\right\rfloor$ and $f_{j} \in S_{j}$ for $1 \leq j \leq\left\lfloor\frac{n+1}{2}\right\rfloor$. With the help of computer again we have obtained the corresponding values for $C_{90}$ and $C_{100}$ as given in Table 1 .

Let $e=u v$ be an arbitrary edge of $C_{10 n}$. We distinguish three typical cases with respect to the position of $u v$. 


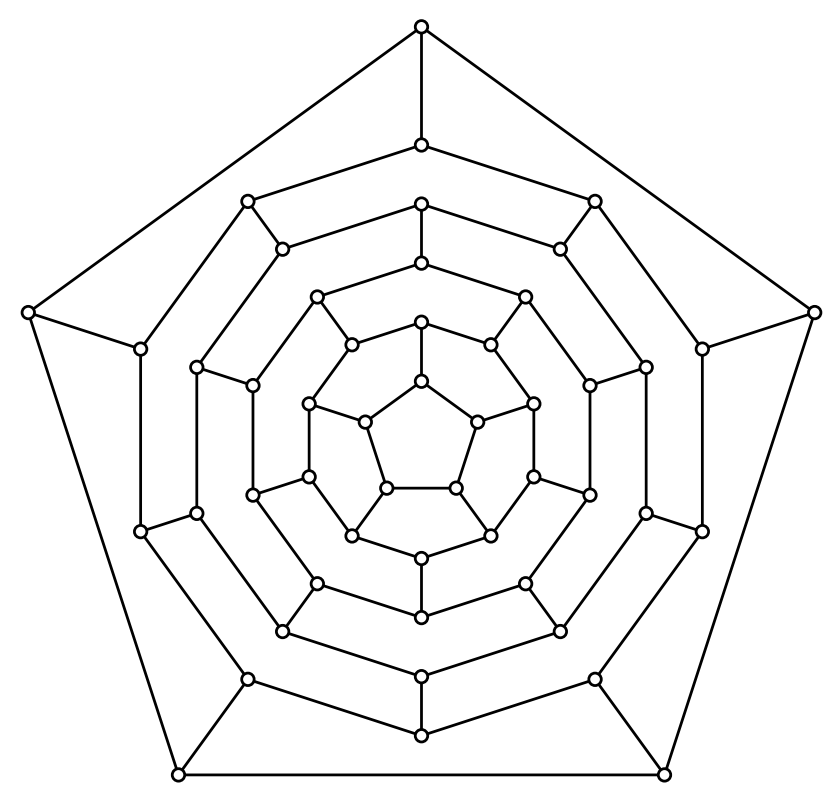

Figure 2. The $C_{50}$ fullerene

\begin{tabular}{|c|c|c|}
\hline$i$ & $N\left(e_{i}\right)$ & $N\left(f_{i}\right)$ \\
\hline \hline 0 & $12 \times 12$ & - \\
\hline 1 & $18 \times 70$ & $9 \times 69$ \\
\hline 2 & $24 \times 64$ & $15 \times 75$ \\
\hline 3 & $32 \times 57$ & $25 \times 65$ \\
\hline 4 & $40 \times 49$ & $35 \times 55$ \\
\hline 5 & - & $45 \times 45$ \\
\hline
\end{tabular}

\begin{tabular}{|c|c|c|}
\hline$i$ & $N\left(e_{i}\right)$ & $N\left(f_{i}\right)$ \\
\hline \hline 0 & $12 \times 12$ & - \\
\hline 1 & $18 \times 80$ & $9 \times 79$ \\
\hline 2 & $24 \times 74$ & $15 \times 85$ \\
\hline 3 & $32 \times 67$ & $25 \times 75$ \\
\hline 4 & $40 \times 59$ & $35 \times 65$ \\
\hline 5 & $50 \times 50$ & $45 \times 55$ \\
\hline
\end{tabular}

Table 1. Values $N(e)$ for the edges of $C_{90}$ (left) and of $C_{100}$ (right)

Suppose first that $u v$ lies within $L_{0}$ or $L_{n+1}$. Then for $n=3$ one can check that $n_{u}(e)=10$, while if $n \geq 4$, then $n_{u}(e)=12$. The latter fact is illustrated in Fig. 3, where the vertices closer to $u$ than to $v$ are colored blue and the vertices closer to $v$ than to $u$ red.

Suppose next that $e=u v \in S_{i}$ for $1 \leq i \leq\left\lfloor\frac{n}{2}\right\rfloor$. Since we will consider the edge $e$ in fullerenes $C_{10 n}$ and in $C_{10(n+1)}$, hence we specify the notation $n_{u}(e)$ to $n_{u}^{(n)}(e)$, meaning that we consider $e$ in $C_{10 n}$. Now we have $N_{u}^{(n+1)}(e)=N_{u}^{(n)}(e)$ and $n_{v}^{(n+1)}(e)=n_{v}^{(n)}(e)+10$ (or vice versa). In addition, when $n$ is odd and $u v \in S_{\left\lfloor\frac{n+1}{2}\right\rfloor}$, then $n_{u}^{(n)}(e)=N_{v}^{(n)}(e)=5 n$.

Assume finally that $u v \in L_{i}$, where $1 \leq i \leq\left\lfloor\frac{n-1}{2}\right\rfloor$, the vertex $u$ is adjacent to some vertex of $L_{i-1}$, and the vertex $v$ is adjacent to some vertex of $L_{i+1}$. Now $n_{u}^{(n+1)}(e)=$ $n_{v}^{(n)}(e)$ and $n_{v}^{(n+1)}(e)=n_{v}^{(n)}(e)+10$. If $n$ is even, then for the edge $u v \in L_{\frac{n}{2}}$ we have $n_{u}^{(n)}(e)=N_{v}^{(n)}(e)=5 n$.

From the above consideration, we conclude that $C_{\mathrm{Sz}}\left(C_{10(n+1)}\right)=C_{\mathrm{Sz}}\left(C_{10 n}\right)+1=n+2$ which completes the inductive argument.

As a consequence of Theorem 4.1, together with a help of computer, we can also determine the Szeged index of the fullerenes $C_{10 n}$. 


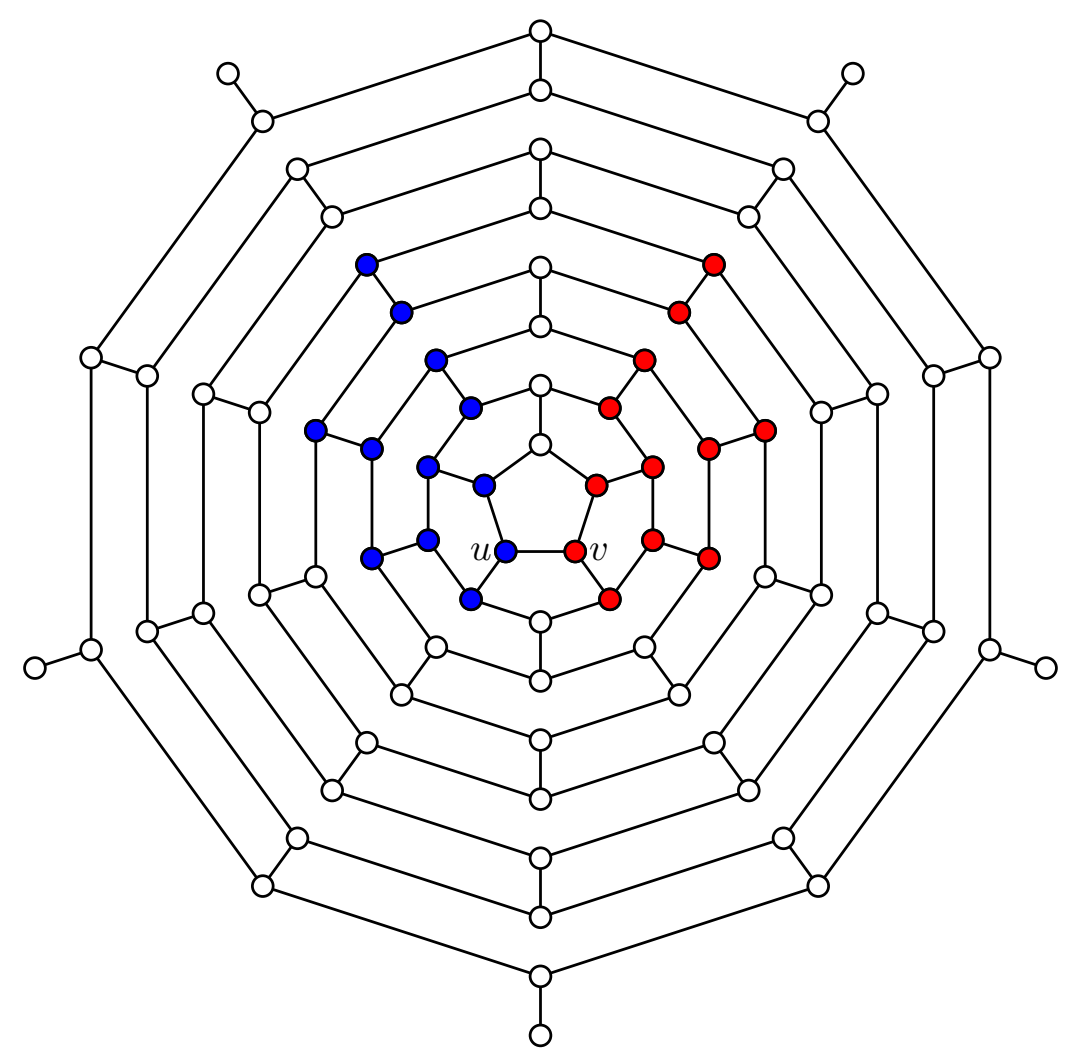

Figure 3. If $e=u v$ lies in $L_{0}$, then $n_{u}(e)=n_{v}(e)=12$

Corollary 4.2. $\mathrm{Sz}\left(C_{30}\right)=6655, \mathrm{Sz}\left(C_{40}\right)=16830, \mathrm{Sz}\left(C_{50}\right)=33545, \mathrm{Sz}\left(C_{60}\right)=58900$, $\mathrm{Sz}\left(C_{70}\right)=93535, \mathrm{Sz}\left(C_{80}\right)=138810$. Moreover, if $n \geq 9$, then

$$
\mathrm{Sz}\left(C_{10 n}\right)=250 n^{3}+3075 n-13800 .
$$

\section{Szeged index versus total eccentricity}

The relation between the Szeged index and the Wiener index has already been well investigated. In [24] it was first proved that $\mathrm{Sz}(G) \geq W(G)$ holds for any connected graph. Moreover, the equality holds if and only if $G$ is a block graph [11,29]. The inequality was in [22] extended by proving that $\operatorname{Sz}(G, w) \geq W(G, w)$ holds for any connected network. In addition, in $[23,26,27,33]$ bounds on $\mathrm{Sz}(G)-W(G)$ and graphs achieving a fixed value of the difference were investigated. In this section we compare the Szeged index with the total eccentricity (cf. [30]) and prove the following result.

Theorem 5.1. If $G$ is a connected graph of order at least 4 , then $\operatorname{Sz}(G) \geq \operatorname{Ecc}(G)$. Moreover, equality holds if and only if $G=P_{4}$.

Proof. It is straightforward to verify that $\operatorname{Sz}(G)>\operatorname{Ecc}(G)$ holds if $G=C_{n}, n \geq 4$ or if $G=P_{n}, n \geq 5$. Moreover, $\operatorname{Sz}\left(P_{4}\right)=\operatorname{Ecc}\left(P_{4}\right)=10$. Hence assume in the rest that $G$ is a connected graph of order at least 4 that is neither a path nor a cycle. In particular, $G$ contains a vertex of degree at least 3 .

Let $v \in V(G)$ and let $v^{\prime}$ be a vertex with $d\left(v, v^{\prime}\right)=\operatorname{ecc}(v)$. Considering a shortest $v, v^{\prime}$-path $P_{v v^{\prime}}$ we infer that $d(v) \geq 1+\cdots+\operatorname{ecc}(v)$. Moreover, since $G$ is not a path, it 
contains at least one vertex that does not lie on $P_{v v^{\prime}}$ and consequently

$$
d(v) \geq(1+\cdots+\operatorname{ecc}(v))+1=\frac{\operatorname{ecc}(v)(\operatorname{ecc}(v)+1)}{2}+1 \geq 2 \operatorname{ecc}(v),
$$

where the latter inequality reduces to $\operatorname{ecc}(v)^{2}+2 \geq 3 \operatorname{ecc}(v)$ which can be easily be verified to hold true.

We have thus shown that $d(v) \geq 2 \operatorname{ecc}(v)$ holds for any vertex $v$ of $G$. Moreover, since $G$ contains at least one vertex, say $w$, of degree at least 3 , by the above argument, but adding 2 instead of 1 , we have $d(w)>2 \operatorname{ecc}(w)$. Therefore

$$
\sum_{v \in V(G)} d(v)>\sum_{v \in V(G)} 2 \operatorname{ecc}(v)=2 \operatorname{Ecc}(G) .
$$

This implies that $W(G)>\operatorname{Ecc}(G)$. Since, as mentioned before the theorem, $\operatorname{Sz}(G) \geq$ $W(G)$ holds for any connected graph $G$, we conclude that $\operatorname{Sz}(G)>\operatorname{Ecc}(G)$.

Theorem 5.1, together by considering the graphs $K_{2}, K_{3}$, and $P_{3}$, yields:

Corollary 5.2. If $G$ is a connected graph with at least one edge, then $\operatorname{Sz}(G)=\operatorname{Ecc}(G)$ if and only if $G \in\left\{K_{3}, P_{4}\right\}$.

To conclude the paper we add that a comparison between the Szeged index and the eccentric connectivity index was done in [10].

Acknowledgment. The second author acknowledges the financial support from the Slovenian Research Agency (research core funding P1-0297, project J1-7110, and project J1-9109).

\section{References}

[1] A. Abiad, B. Brimkov, A. Erey, L. Leshock, X. Martínez-Rivera, S. O, S.-Y. Song and J. Williford, On the Wiener index, distance cospectrality and transmission-regular graphs, Discrete Appl. Math. 230, 1-10, 2017.

[2] Y. Alizadeh, Szeged dimension and $P I_{v}$ dimension of composite graphs, Iranian J. Math. Sci. Inform. 13, 45-57, 2018.

[3] Y. Alizadeh, V. Andova, S. Klavžar and R. Škrekovski, Wiener dimension: fundamental properties and (5,0)-nanotubical fullerenes, MATCH Commun. Math. Comput. Chem. 72, 279-294, 2014.

[4] Y. Alizadeh and S. Klavžar, Complexity of topological indices: The case of connective eccentric index, MATCH Commun. Math. Comput. Chem. 76, 659-667, 2016.

[5] Y. Alizadeh and S. Klavžar, On graphs whose Wiener complexity equals their order and on Wiener index of asymmetric graphs, Appl. Math. Comput. 328, 113-118, 2018.

[6] V. Andova, T. Došlić, M. Krnc, B. Lužar and R. Škrekovski, On the diameter and some related invariants of fullerene graphs, MATCH Commun. Math. Comput. Chem. 68, 109-130, 2012.

[7] M. Arockiaraj, J. Clement and K. Balasubramanian, Analytical expressions for topological properties of polycyclic benzenoid networks, J. Chemometrics, 30, 682-697, 2016.

[8] M. Arockiaraj, J. Clement and A.J. Shalini, Variants of the Szeged index in certain chemical nanosheets, Canad. J. Chem. 94, 608-619, 2016.

[9] D. Buset, Orbits on vertices and edges of finite graphs, Discrete Math. 57, 297-299, 1985.

[10] K.Ch. Das and M.J. Nadjafi-Arani, Comparison between the Szeged index and the eccentric connectivity index, Discrete Appl. Math. 186, 74-86, 2015. 
[11] A.A. Dobrynin and I. Gutman, Solving a problem connected with distances in graphs, Graph Theory Notes N. Y. 28, 21-23, 1995.

[12] R.C. Entringer, D.E. Jackson and D.A. Snyder, Distance in graphs, Czechoslovak Math. J. 26, 283-296, 1976.

[13] M. Goubko and O. Miloserdov, Simple alcohols with the lowest normal boiling point using topological indices, MATCH Commun. Math. Comput. Chem. 75, 29-56, 2016.

[14] S. Gupta, M. Singh and A.K. Madan, Connective eccentricity index: a novel topological descriptor for predicting biological activity, J. Mol. Graph. Model. 18, 18-25, 2000.

[15] I. Gutman, A formula for the Wiener number of trees and its extension to graphs containing cycles, Graph Theory Notes N. Y. 27, 9-15, 1994.

[16] I. Gutman and B. Furtula (Eds.), Novel Molecular Structure Descriptors-Theory and Applications I, Univ. Kragujevac, Kragujevac, 2010.

[17] I. Gutman and B. Furtula (Eds.), Novel Molecular Structure Descriptors-Theory and Applications II, Univ. Kragujevac, Kragujevac, 2010.

[18] I. Gutman, K. Xu and M. Liu, A congruence relation for Wiener and Szeged indices, Filomat, 29, 1081-1083, 2015.

[19] M. Imran, A.Q Baig and H. Ali, On molecular topological properties of hex-derived networks, J. Chemometrics, 30, 121-129, 2016.

[20] P.V. Khadikar, S. Karmarkar, V.K. Agrawal, J. Singh, A. Shrivastava, I. Lukovits and M.V. Diudea, Szeged index - applications for drug modeling, Lett. Drug Des. Discov. 2, 606-624, 2005.

[21] S. Klavžar, D. Azubha Jemilet, I. Rajasingh, P. Manuel and N. Parthiban, General Transmission Lemma and Wiener complexity of triangular grids, Appl. Math. Comput. 338, 115-122, 2018.

[22] S. Klavžar and M.J. Nadjafi-Arani, Wiener index versus Szeged index in networks, Discrete Appl. Math. 161, 1150-1153, 2013.

[23] S. Klavžar and M.J. Nadjafi-Arani, Improved bounds on the difference between the Szeged index and the Wiener index of graphs, European J. Combin. 39, 148-156, 2014.

[24] S. Klavžar, A. Rajapakse and I. Gutman, The Szeged and the Wiener index of graphs, Appl. Math. Lett. 9, 45-49, 1996.

[25] S. Li and H. Zhang, Proofs of three conjectures on the quotients of the (revised) Szeged index and the Wiener index and beyond, Discrete Math. 340, 311-324, 2017.

[26] M.J. Nadjafi-Arani, H. Khodashenas and A.R. Ashrafi, On the differences between Szeged and Wiener indices of graphs, Discrete Math. 311, 2233-2237, 2011.

[27] M.J. Nadjafi-Arani, H. Khodashenas and A.R. Ashrafi, Graphs whose Szeged and Wiener numbers differ by 4 and 5, Math. Comput. Modelling, 55, 1644-1648, 2012.

[28] F. Shafiei, M. Pashm Froush and F. Dialamehpour, QSPR study on benzene derivatives to some physicochemical properties by using topological indices, Iranian J. Math. Chem. 7, 93-110, 2016.

[29] S. Simić, I. Gutman and V. Baltić, Some graphs with extremal Szeged index, Math. Slovaca, 50, 1-15, 2000.

[30] H. Smith, L. Székely and H. Wang, Eccentricity sums in trees, Discrete Appl. Math. 207, 120-131, 2016.

[31] R. Todeschini and V. Consonni, Molecular Decriptors for Chemoinformatic, WileyVCH, Weinheim, 2009.

[32] K. Xu, K.Ch. Das and H. Liu, Some extremal results on the connective eccentricity index of graphs, J. Math. Anal. Appl. 433, 803-817, 2016.

[33] H. Zhang, S. Li and L. Zhao, On the further relation between the (revised) Szeged index and the Wiener index of graphs, Discrete Appl. Math. 206, 152-164, 2016. 